Cebollero, P.; Zambom-Ferraresi, F.; Hueto, J.; Hernández, M.; Cascante, J.; Antón, M.M. (2021) Effects of a Physical Activity Programme on the Muscle Function in Patients with COPD. Revista Internacional de Medicina y Ciencias de la Actividad Física y el Deporte vol. 21 (82) pp. 389-401 Http://cdeporte.rediris.es/revista/revista82/artefectos1259.htm

DOI: https://doi.org/10.15366/rimcafd2021.82.012

\title{
ORIGINAL
}

\section{EFECTOS DE REALIZAR ACTIVIDAD FÍSICA EN LA FUNCIÓN MUSCULAR EN EPOC}

\section{EFFECTS OF A PHYSICAL ACTIVITY PROGRAMME ON THE MUSCLE FUNCTION IN PATIENTS WITH COPD}

\author{
Cebollero, P. '; Zambom-Ferraresi, F. ${ }^{2}$; Hueto, J.'; Hernández, M.'; Cascante, \\ J. $^{1}$ y Antón, M.M. ${ }^{4}$ \\ ${ }^{1}$ Neumólogos, Complejo Hospitalario de Navarra (España) pilar.cebollero.rivas@cfnavarra.es, \\ javierhuepede@gmail.com, ja.cascante.rodrigo@cfnavarra.es \\ 2 Investigador, Navarrabiomed (España) fabriciogigante@hotmail.com \\ ${ }^{3}$ Neumóloga, Clínico de Zaragoza (España) mhernandezb@salud.aragon.es \\ ${ }^{4}$ Titular de Universidad, Universidad Pública de Navarra (España) milagros.anton@unavarra.es
}

\section{AGRADECIMIENTOS}

Este estudio se financió con una ayuda del Plan Nacional I+D+i 2008-20011, Gobierno de España (ref: DEP2011-30042).

No se ha tenido financiación del aparataje utilizado.

Código UNESCO / UNESCO Code: 320508 (Enfermedades Pulmonares / Pulmonary Diseases)

Clasificación del Consejo de Europa / Council of Europe Classification: 17. Otras: Actividad Física y Salud / Others: Physical Activity and Health

Recibido 21 de junio de 2019 Received June 21, 2019

Aceptado 29 de febrero de 2020 Accepted February 29, 2020

\section{RESUMEN}

El objetivo de este estudio fue examinar los efectos de un programa de actividad física (AF) de andar en la función muscular en pacientes con EPOC (Enfermedad Pulmonar Obstructiva Crónica), con un seguimiento de 12 meses. Se reclutaron 44 hombres (70,3 $\pm 6,7$ años) diagnosticados de EPOC moderado-severo. El grupo intervención realizó un programa de actividad física (GAF) y el grupo control (GCO) siguió su tratamiento estándar. Se midió la fuerza máxima (1RM) del miembro inferior y superior, la potencia muscular del miembro inferior $(50 \%$ y $70 \% 1 \mathrm{RM}$ ) y la actividad física, antes y después de 12 meses. A los 12 meses, incremento un $8 \%(P<0,01)$ la $1 R M$ del miembro inferior en GAF, sin cambios en GCO. La potencia muscular al 50\%1RM incrementó un $12 \%$ en GAF, 
disminuyendo un $9 \%$ in $\mathrm{GCO}(\mathrm{P}<0,05)$. Un programa de AF incrementa la fuerza y preserva la potencia muscular del miembro inferior.

PALABRAS CLAVE: Enfermedad Pulmonar Obstructiva Crónica, actividad física, fuerza muscular, potencia muscular, disfunción muscular periférica.

\section{ABSTRACT}

The purpose of this study was to examine the effects of a 12-month physical activity (PA) programme consisting in walking on muscle function in patients with COPD (Chronic Obstructive Pulmonary Disease). Forty-four men (70.3 \pm 6.7 years old) diagnosed with moderate-severe COPD were recruited. The intervention group (PAG) completed a physical activity programme and the control group (COG) followed their standard treatment. Upper- and lower-limb maximum strength (1RM), lower-limb muscle power (50\% and 70\% 1RM) and physical activity were measured before and after 12 months. After 12 months, lower-limb maximum strength increased by $8 \%$ in the PAG $(P<0.01)$, while it did not change in the COG. Lower-limb muscle power at 50\% $1 \mathrm{RM}$ increased by $12 \%$ in the PAG, while it decreased by $9 \%$ in the COG $(P<0.05)$. A physical activity programme increased muscle strength and preserved muscle power of the lower limb.

KEY WORDS: chronic obstructive pulmonary disease, physical activity, muscle strength, muscle power, skeletal muscle dysfunction.

\section{INTRODUCCIÓN}

La disfunción muscular periférica es una consecuencia sistémica de las enfermedades respiratorias crónicas, entre las que se encuentra la Enfermedad Pulmonar Obstructiva Crónica (EPOC), incrementando la prevalencia con la severidad de la enfermedad (Seymor et al., 2010). La disfunción muscular periférica en personas con EPOC se caracteriza por alteraciones a nivel funcional, metabólico y morfológico del músculo esquelético de las extremidades superiores e inferiores (Nyberg et al., 2015; Maltais et al., 2014). A nivel funcional, se ha observado una disminución de la fuerza máxima y resistencia muscular y una mayor fatigabilidad muscular en pacientes con EPOC en comparación con personas sanas (Bernard et al., 1998; Coronell et al., 2004). Reducciones en la masa muscular (Marquis et al., 2002) y en la fuerza muscular (Swallow et al., 2007) son predictores de mortalidad en estos pacientes. Clínicamente, esta disfunción muscular periférica se asocia a la función pulmonar (Cebollero et al., 2017), a una intolerancia al ejercicio físico (Seymor et al., 2010; Gosselink et al., 1996), una mayor disnea (Seymor et al., 2010), peor calidad de vida (Mostert et al., 2000) y una mayor utilización de recursos sanitarios (Decramer et al., 1997).

El proceso de envejecimiento está asociado con una disminución de la masa muscular, de la fuerza máxima y de la potencia muscular de las extremidades (Izquierdo et al., 1999). La fuerza máxima disminuye a partir de los 50 años (12- 
15\%/década)(Hurley, 1995; Hunter et al., 2004). Se ha observado que el envejecimiento está asociado con una mayor dificultad para caminar, subir escalera, agacharse y subir escaleras en la población española (LeirósRodríguez et al., 2018). En personas de edad avanzada, la potencia muscular (producto de la fuerza y de la velocidad) de las extremidades inferiores a velocidades elevadas podría ser más importante que la fuerza máxima para la realización de actividades de la vida diaria como andar y subir escaleras (Bassey et al., 19992; Bean et al., 2002; Bean et al., 2003). En pacientes con EPOC de edad avanzada, se ha observado una asociación entre la potencia muscular de las extremidades inferiores con la función respiratoria (Cebollero et al., 2017) y la tolerancia al ejercicio físico y entre la potencia muscular y la actividad física (Hernández et al., 2017).

Esta disfunción muscular es consecuencia mayoritariamente de la inactividad física, secundaria a la disnea, que produce un decondicionamiento físico en los pacientes con EPOC (Hernández et al., 2017; Waschki et al., 2015). Se ha demostrado que el entrenamiento de fuerza muscular, componente fundamental de los programas de rehabilitación respiratoria, mejora la tolerancia al ejercicio, la fuerza muscular, la disnea, la fatiga y, la calidad de vida (Zambom-Ferraresi et al., 2015). Por lo tanto, es una intervención terapéutica fundamental para el tratamiento de la disfunción muscular en pacientes con EPOC. La actividad física en personas mayores de 50 años está asociada al estado de salud percibido (Lera-López et al., 2017), así como a una mejor condición física (Moral-García et al., 2019) y estado de salud (Salinas Martínez et al., 2010). Sin embargo, es menos conocido si un programa de actividad física puede prevenir o mejorar la función muscular periférica de los pacientes con EPOC.

Por lo tanto, el objetivo de este estudio fue examinar los efectos de un programa de actividad física de andar en la fuerza máxima y la potencia muscular en pacientes con EPOC, con un seguimiento de 12 meses. La hipótesis fue que en pacientes con EPOC, un programa de actividad física de andar preservaría los niveles de fuerza máxima y de potencia muscular de las extremidades inferiores.

\section{MATERIAL Y MÉTODOS}

\section{Participantes}

Este no es un estudio aleatorizado. Se compararon dos grupos: un grupo intervención y un grupo de referencia o grupo control. Se incluyeron un total de 44 pacientes procedentes de las consultas de los servicios de neumología con los siguientes criterios: 1) varones diagnosticados con EPOC moderado-severo, un ratio de $\mathrm{FEV}_{1} / \mathrm{FVC}<0.70$ y $\mathrm{FEV}_{1}$ entre el $30 \%-80 \%$ post-broncodilatado respecto a los varones de referencia (estadio II-III según GOLD (Vestbo et al., 2013)); 2) disnea grado 2-3 medido con la escala de disnea modifica del British Medical Council (mMRC y, 3) con una edad superior a 50 años. Los criterios de exclusión fueron: 1) fumadores activos, 2) exacerbación en los 3 últimos meses, 3) cardiopatía inestable, y 4) neoplasia o cualquier condición neuromuscular, esquelética o artrítica que le pudiera limitar la tolerancia al ejercicio físico. Todos los pacientes realizaron una espirometría, pletismografía, el test de 6 minutos marcha (T6MM) y, el cuestionario de evaluación de la EPOC que mide el impacto 
de la enfermedad (CAT). Se cumplieron los requisitos de la Declaración de Helsinki y el Comité Ética, Experimentación Animal, y Bioseguridad de la Universidad Pública de Navarra ( $N^{\circ}$ de aprobación: PI-002/11) aprobó el estudio. Los datos personales fueron codificados para la realización del análisis de datos. Todos los participantes dieron su consentimiento informado.

\section{Programa de intervención}

Los participantes fueron medidos al inicio (basal) y a los 12 meses de seguimiento. El grupo intervención ( $\mathrm{N}=32$; $\mathrm{GAF}$ ) realizó un programa de actividad física que consistía en andar o paseos, durante 12 meses de seguimiento. Se les daba la indicación de que debían de andar al menos 3 días a la semana, un paseo de al menos 30 minutos. Todos los pacientes cumplimentaban un registro diario de síntomas y exacerbaciones. Mensualmente se les hacía una llamada telefónica en la que se preguntaba la media de cumplimentación mensual de las indicaciones dadas.

El grupo control o de cuidado estándar ( $\mathrm{N}=12 ; \mathrm{GCO})$ siguió las recomendaciones estándar sobre actividad física dadas en la consulta de neumología. Este grupo no fue formado con pacientes que hubiesen rechazado participar en el programa de actividad física.

\section{Medidas de función muscular}

Valoración de la fuerza máxima de las extremidades inferiores. La fuerza máxima en el ejercicio de extensión bilateral de rodillas fue medida con el test de una repetición máxima (1RM) en una máquina de fuerza comercial (Technogym, Gambettola, Italia) y fue definida como el peso máximo levantado en el ejercicio de extensión bilateral de rodillas. El día del test, los participantes realizaron un calentamiento que consistió en andar 5-min y ejercicios de estiramientos. Además, realizaron varias contracciones para el ejercicio de extensión de rodillas. Después, se utilizaron cuatro a cinco intentos para determinar el 1RM. Los intentos fueron separados por descansos de 2-min entre intentos. La 1RM fue definida como el peso máximo levantado en un solo intento.

Valoración de la potencia muscular de las extremidades inferiores. Después de medir 1RM, la potencia muscular en el ejercicio de prensa de piernas fue medida. La potencia muscular de los extensores de las piernas (W) y de la cadera fue medida durante la fase concéntrica del ejercicio de prensa bilateral de piernas utilizando el peso $(\mathrm{kg})$ que correspondía al $50 \%$ y al $70 \%$ de $1 \mathrm{RM}$. Se les indicó a los participantes que desplazarán el peso lo más rápidamente posible. Se realizó dos intentos con cada peso, con 2-min de recuperación entre intentos. El mejor intento en cada peso, se utilizó para los análisis.

Valoración de la fuerza máxima de las extremidades superiores. La fuerza máxima (1RM) de las extremidades superiores fue medido en el ejercicio de pectoral sentado y en el dorsal sentado en una máquina de fuerza comercial. La carga máxima fue determinada en cuatro a cinco intentos, separados por 
descansos de 2-min entre intentos. La 1RM fue definida como el último intento aceptado como válido, ejecutado con el peso máximo.

\section{Análisis estadístico}

Las variables se expresaron como media \pm desviación estándar (SPSS versión 20.0, IBM Corporation, Armonk, NY, USA). Se comprobó la normalidad de la distribución de las variables con el test de Shapiro-Wilk. Los valores basales se compararon entre grupos con la t-de Student o con el test de U Mann-Whitney. La comparación de tratamiento (efecto tiempo) dentro de los grupos se realizó con la t-de Student para variables paramétricas o con el test de Wilcoxon. El valor de $\mathrm{P}$ fue establecido significativo en $<0,05$.

\section{RESULTADOS}

Las características basales de la población se muestran en la Tabla 1. No se observaron diferencias significativas entre los grupos al inicio del estudio en ninguna de las variables. Se reclutaron un total de 44 pacientes, procedentes de la consulta de neumología: 32 en el grupo de actividad física (GAF) y 12 en el grupo control (GCO); las pérdidas en el GAF fueron de 5 abandonos y 1 fallecimiento y, en el GCO, 3 abandonos. Los resultados de disnea, CAT, T6MM y el número de pasos al día (actividad física) se han publicado previamente (Cebollero et al., 2018).

\begin{tabular}{lllllll}
\hline Variable & GAF & \multicolumn{3}{c}{ GCO } & & \multicolumn{1}{c}{ Valor } \\
& $\mathrm{N}=32$ & & $\mathrm{~N}=12$ & & $\boldsymbol{P}$ \\
\hline Edad (años) & 69,9 & $\pm 7,2$ & 71,0 & $\pm 5,2$ & 0,8 \\
IMC (kg/m²) & 28,4 & $\pm 4,5$ & 28,5 & $\pm 7,1$ & 0,8 \\
FVC (\%) & 77,0 & $\pm 12,2$ & 75,7 & \pm 21 & 0,6 \\
FEV (\%) & 51,5 & $\pm 14,2$ & 50,2 & $\pm 12,5$ & 0,9 \\
FEV $/$ /FVC (\%) & 47,5 & $\pm 11,5$ & 48,3 & $\pm 9,1$ & 0,8 \\
T6MM (m) & 478,3 & $\pm 66,5$ & 473,5 & $\pm 79,5$ & 0,8 \\
Disnea (mMRC, puntos) & 2,1 & $\pm 0,3$ & 2,0 & \pm 0 & 0,1 \\
Potencia muscular 50\% 1RM (W) & 573,7 & $\pm 264,9$ & 585,7 & $\pm 205,4$ & 0,7 \\
Potencia muscular 70\% 1RM (W) & 568,0 & $\pm 259,9$ & 582,5 & $\pm 203,4$ & 0,7 \\
1-RM Extensión rodillas (kg) & 73,0 & $\pm 20,8$ & 69,4 & $\pm 17,6$ & 0,6 \\
1-RM Pectoral sentado (kg) & 55,5 & $\pm 15,7$ & 55,1 & $\pm 12,3$ & 0,9 \\
1-RM Dorsal (kg) & 53,4 & $\pm 12,2$ & 52,8 & $\pm 13,9$ & 0,9 \\
\hline
\end{tabular}

GAF: grupo de actividad física; GCO: grupo control; IMC: índice de masa corporal; FVC: capacidad vital forzada; FEV 1 : volumen espirado forzado en el primer segundo; T6MM: test de 6 minutos marcha; mMRC: escala de disnea modifica del British Medical Council; 1RM: una repetición máxima. Los datos son expresados en media \pm desviación estándar.

La fuerza máxima de las extremidades inferiores, en el ejercicio de extensión de rodillas, incremento un $8,5 \pm 9,9 \%$ en el GAF (desde $78 \pm 18$ a 84,5 \pm 19,5 kg; $\mathrm{P}=0,004$ ), mientras que no se observó ninguna mejora en el GCO (desde 68,1 \pm 11,3 a $67,1 \pm 9,5 \mathrm{~kg} ; \mathrm{P}=0,6$ ) después de 12 meses de seguimiento (Figura 1A). 
La mejora de la fuerza máxima de las extremidades superiores en el ejercicio de pectoral sentado de un 10,8 $\pm 16,3 \%$ en el GAF (desde 58,7 $\pm 14,6$ a $64,5 \pm 15,9$ $\mathrm{kg} ; \mathrm{P}=0,003)$ fue significativamente superior $(P=0,027)$ que la observada de $-3,4$ $\pm 11,4 \%$ en el GCO (desde 56,9 $\pm 8,1$ a $54,6 \pm 8,7 \mathrm{~kg} ; \mathrm{P}=0,4$ ) (Figura 1B). La fuerza máxima de las extremidades superiores en el ejercicio de dorsal sentado incremento en el GAF (desde $55,2 \pm 12,8$ a $58,1 \pm 11,8 \mathrm{~kg} ; \mathrm{P}=0,004$ ), mientras no se observó ninguna mejora en el GCO (desde $55 \pm 11,2$ a $55 \pm 10 \mathrm{~kg} ; \mathrm{P}=1,0$ ) al finalizar los 12 meses de seguimiento.

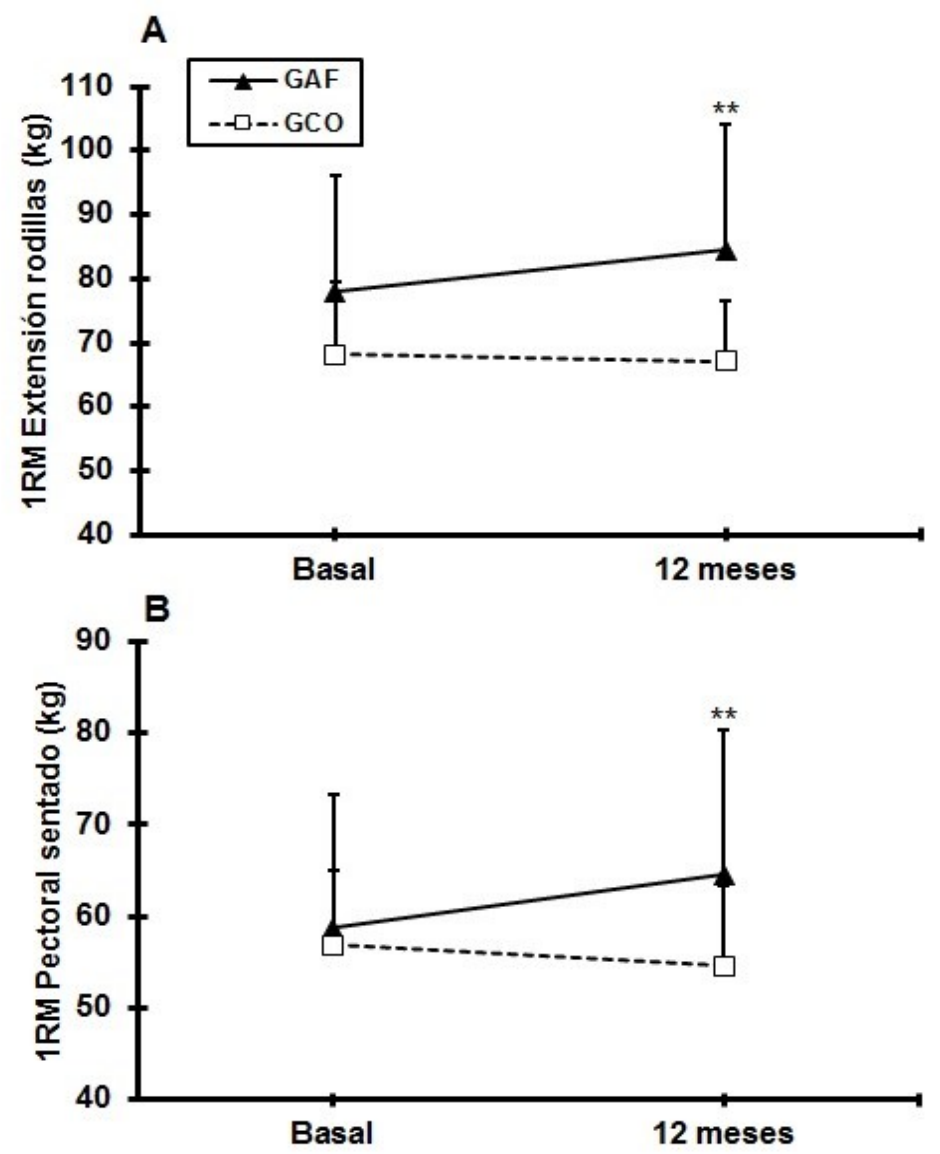

Figura 1. Fuerza máxima (1RM) de las extremidades inferiores $(A)$ y de las extremidades superiores (B) antes y después de 12 meses de seguimiento en el grupo de actividad física (GAF) y grupo control (GCO). $\mathrm{N}=20$ completaron el test en el GAF y $\mathrm{N}=7$ completaron el test en el GCO. Nivel de significación: ${ }^{* \star} \mathrm{P}<0,01$ basal-12 meses dentro del mismo grupo.

Después de 12 meses de seguimiento, la mejora de la potencia muscular de las extremidades inferiores al $50 \%$ de $1 \mathrm{RM}$, de $12 \pm 49,6 \%$ en el GAF (desde 659,1 $\pm 287,1$ a $684,7 \pm 247,3 \mathrm{~W} ; \mathrm{P}=0,8)$ fue significativamente superior $(\mathrm{P}=0,014)$ que la observada de $-9,2 \pm 6,1 \%$ en el GCO (desde $568 \pm 79,7$ a $513,3 \pm 57,6 \mathrm{~W}$; $\mathrm{P}=0,026$ ) (Figura 2A). Ni el GAF ni el GCO modificaron la potencia muscular al $70 \%$ de 1 RM después de 12 meses de seguimiento. No se observaron diferencias significativas en la potencia muscular al $70 \%$ de $1 \mathrm{RM}$ de las extremidades inferiores entre los grupos después de dicho periodo de seguimiento (Figura 2B). 


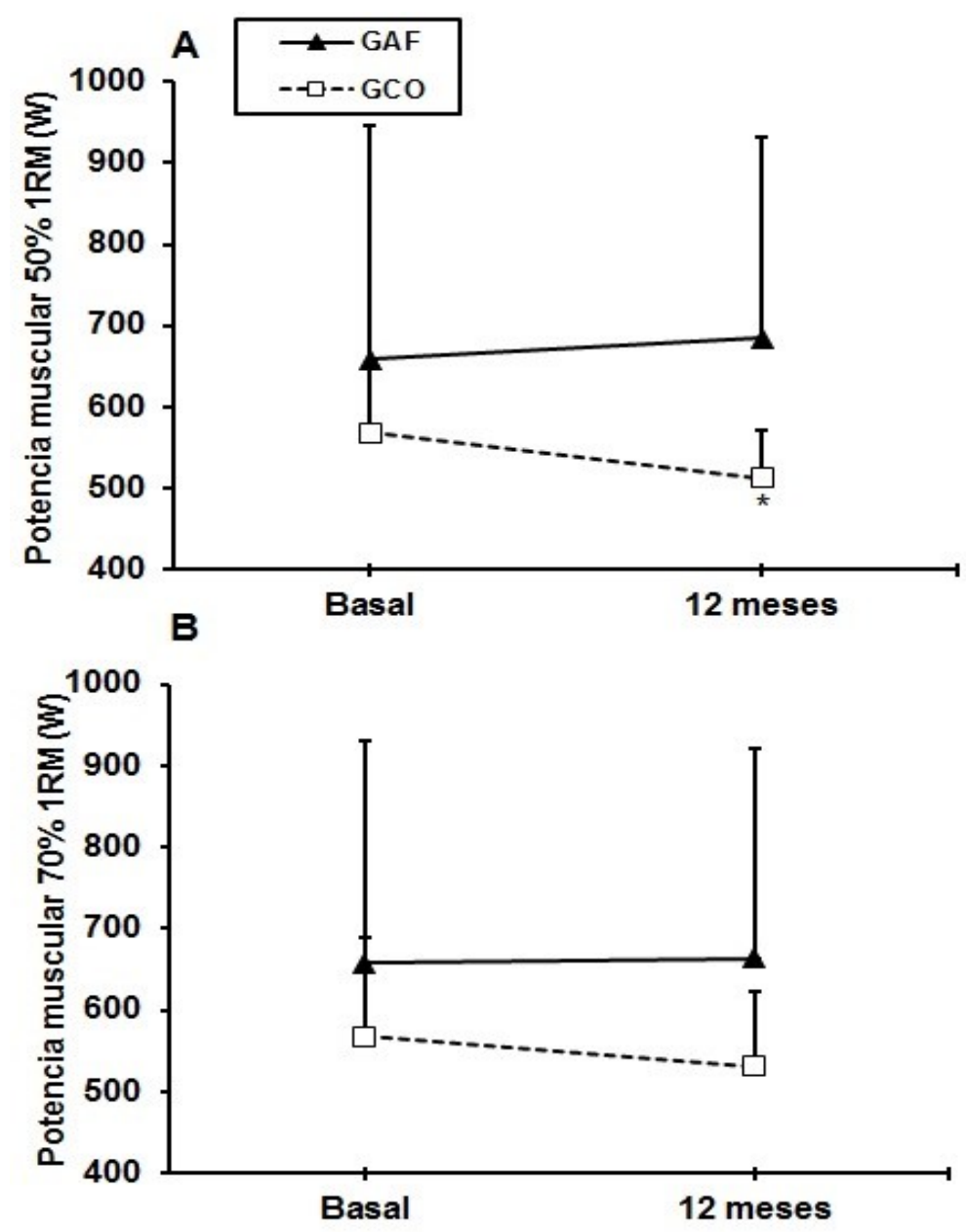

Figura 2. Potencia muscular de las extremidades inferiores al $50 \% 1 \mathrm{RM}(\mathrm{A})$ y al $70 \%$ $1 R M(B)$ antes y después de 12 meses de seguimiento en el grupo de actividad física (GAF) y grupo control (GCO). $\mathrm{N}=18$ completaron el test en el GAF y $\mathrm{N}=6$ completaron el test en el GCO. Nivel de significación: * $P<0,05$ basal-12 meses dentro del mismo grupo.

\section{DISCUSIÓN}

Los resultados más importantes de este estudio fueron que 12 meses de un programa de actividad física, que consistía en caminar al menos 30 minutos, 3 días a la semana, incrementó la fuerza máxima de las extremidades inferiores y superiores $\mathrm{y}$, además, preservo los valores de potencia muscular del miembro inferior en pacientes con EPOC; frente a la disminución de la misma en el grupo control, después de 12 meses de seguimiento.

La disfunción muscular periférica en las personas con EPOC se ha postulado como uno de los efectos sistémicos extrapulmonares más importantes, ya que se asocia a la mortalidad, calidad de vida e intolerancia a realizar las actividades de la vida diaria (Marquis et al., 2002; Decramer et al., 1997). Esta disfunción muscular periférica se caracteriza por una disminución de la fuerza máxima y la masa muscular de las extremidades inferiores (Bernard et al., 1998; Gosker et al., 2003). Previos estudios en personas con EPOC (Beijers et al., 2017, van de Bool et al., 2017) han observado un incremento de la fuerza máxima del miembro 
inferior después de un programa de ejercicio físico de resistencia aeróbica y fuerza muscular. Sin embargo, el ejercicio físico no es sinónimo de actividad física. Mientras que el ejercicio físico se define como un subtipo de actividad física que es planeada, estructurada, repetitiva con el objetivo de mejorar o mantener la aptitud física; la actividad física se define como cualquier movimiento llevado a cabo por la musculatura esquelética que origine un gasto de energía (Caspersen et al., 1985). En el presente estudio, la fuerza máxima del miembro inferior en el ejercicio de extensión de rodillas incremento un $8 \%$ después de realizar el programa de actividad física, mientras que no mejoro en el grupo control. Cebollero et al. (2018) observaron previamente que después de 12 meses de seguimiento; el número de pasos al día se mantenía en el grupo que realizaba un programa de actividad física, sugiriendo que el nivel de actividad física se había mantenido durante 12 meses (Cebollero et al., 2018). El hecho que los niveles de actividad física permanecieron estables en el grupo que realizó el programa de actividad física durante los 12 meses de seguimiento podría ser la explicación de la mejora de la fuerza máxima.

Además, uno de los hallazgos observados en el presente estudio es que el grupo que realizó el programa de actividad física incrementó un 10\% la fuerza máxima de las extremidades superiores. En base a nuestros resultados, no es posible identificar los mecanismos fisiológicos responsables de la mejora de la fuerza máxima del miembro superior después de realizar un programa de andar. Sin embargo, los hallazgos podrían ser explicados por el mantenimiento del nivel de actividad física durante los 12 meses, que les podría haber permitido realizar un mayor número de actividades de la vida diaria y, por consiguiente, incrementar la fuerza de las extremidades superiores. En concordancia con los resultados del presente estudio, Garcia-Aymerich et al. (2009) observaron que las personas con EPOC con niveles de actividad física más elevados fueron lo que presentaron valores más altos de la fuerza máxima de los músculos espiratorios. Por lo tanto, se podría concluir que un programa de actividad física, que consistía en andar, al menos 3 días a la semana, con una duración de 30 minutos es una estrategia sencilla para mejorar la disfunción muscular periférica de las extremidades superiores e inferiores en los pacientes con EPOC.

La disminución de la fuerza muscular y la potencia muscular durante el proceso de envejecimiento ha sido atribuida a una pérdida de masa muscular mediada por una pérdida de fibras musculares y del tamaño de las mismas, así como la reducción de actividad física que se observa con el envejecimiento (Hunter et al., 2004). La pérdida de potencia muscular del miembro inferior ha emergido como una variable más significativa incluso que la fuerza máxima del miembro inferior para el mantenimiento de la capacidad funcional de las personas con EPOC (Hernández et al., 2017). En personas de edad avanzada, la potencia muscular a altas velocidades de las extremidades inferiores es un factor más importante en la realización de las actividades de la vida diaria que la fuerza máxima (Bassey et al., 1992; Cadore et al., 2014; Casas-Herrero et al., 2013). Sin embargo, en lo que nosotros conocemos existen muy pocos estudios que hayan examinado los efectos de un programa de actividad física durante un periodo de 12 meses en la potencia muscular de las extremidades inferiores en pacientes con EPOC. Los hallazgos del presente estudio mostraron que la potencia muscular del miembro inferior se mantuvo en el grupo que realizaba el programa 
de actividad física, mientras que en el grupo control disminuía significativamente después de 12 meses de seguimiento. Por lo tanto, los resultados del presente estudio sugieren que un programa de actividad física, que consistía en caminar o, al menos 3 días a la semana, con una duración de 30 minutos mantiene la potencia muscular del miembro del inferior en los pacientes con EPOC. Una sugerencia práctica de los resultados de estudio es la necesidad de incluir un programa de potencia muscular para las extremidades inferiores en personas con EPOC dentro del programa de actividad física. El programa de potencia muscular del miembro inferior consistiría en un ejercicios de fuerza del miembro inferior, realizados a una intensidad moderada (40-60\% 1RM)(Izquierdo et al., 1999), realizado 2 veces por semana, 2-3 series, entre 8-10 repeticiones, que comenzaría al 40\% 1RM y se progresaría hasta el $60 \%$ de 1RM. Durante la ejecución de los ejercicios se le indicaría que lo debería de realizar en lo más rápidamente posible. Estas recomendaciones que están realizadas en personas sanas de edad avanzada, deberían de ser consideradas en futuros estudios de investigación en personas con EPOC.

El presente estudio tiene varias limitaciones metodológicas. La primera, es un estudio comparando un grupo de tratamiento con un grupo de referencia, no es aleatorizado. El grupo control fue seleccionado con posterioridad, siguió las recomendaciones estándar sobre actividad física recibidas en la consulta de neumología, y en ningún caso se formó con pacientes que hubiesen rechazado participar en el programa. A pesar de la no aleatorización, los dos grupos fueron equivalentes en las mediciones basales. La segunda, la muestra es pequeña. Por último, todos los participantes fueron varones, y por lo tanto, los resultados no se pueden extrapolar a mujeres. En España, la prevalencia de personas con EPOC es superior en varones (15,1\%) que en mujeres (5,6\%)(Miravitlles et al., 2009). Por último, no se midió la masa muscular en este estudio que podría haber explicado los cambios observados en la fuerza y potencia muscular después de la intervención. Una fortaleza de este estudio es la adherencia de los pacientes al estudio, a pesar de los 12 meses de seguimiento.

\section{CONCLUSIONES}

En conclusión, 12 meses de un programa de actividad física basado en andar, al menos 30 minutos al día, 3 días a la semana, es efectivo para incrementar la fuerza máxima del miembro inferior y superior. Además, preserva los niveles de potencia muscular del miembro inferior. Este tipo de iniciativa, que es sencilla y fácil de aplicar como un programa de andar, debería estar incluida en la práctica clínica habitual para prevenir y/o mejorar la disfunción muscular periférica en los pacientes con enfermedad pulmonar obstructiva crónica.

\section{REFERENCIAS BIBLIOGRÁFICAS}

Bassey, E. J., Fiatarone, M. A., O'Neill, E. F., Kelly, M., Evans, W. J., \& Lipsitz, L. A. (1992). Leg extensor power and functional performance in very old men and women. Clinical Science (London), 82, 321-327. https://doi.org/10.1042/cs0820321 
Bean, J.F., Kiely, D.K., Herman, S., Leveille, S.G., Mizer, K., . . Fielding, R.A. (2002). The relationship between leg power and physical performance in mobility-limited older people. J Am Geriatr Soc, 50(3), 461-467.

https://doi.org/10.1046/j.1532-5415.2002.50111.x

Bean, J.F., Leveille, S.G., Kielyl, D.K., Bandinelli, S., Guralnik, J.M. \& Ferrucci, L. (2003). A comparison of leg power and leg strength within the InCHIANTI study: which influences mobility more? J Gerontol A Biol Sci Med Sci, 58(8), 728-733. https://doi.org/10.1093/gerona/58.8.M728

Beijers, R.J.H.C.G., van de Bool, C., van den Borst, B., Franssen, F.M.E., Wouters, E.F.M., \& Schols, A.M.W.J. (2017). Normal Weight but Low Muscle Mass and Abdominally Obese: Implications for the Cardiometabolic Risk Profile in Chronic Obstructive Pulmonary Disease. $J$ Am Med Dir Assoc, 18(6):533-538. https://doi.org/10.1016/j.jamda.2016.12.081

Bernard, S., Leblanc, P., Whittom, F., Carrier, G., Jobin, J., Belleau, R., \& Maltais, F. (1998). Peripheral muscle weakness in patients with chronic obstructive pulmonary disease. Am J Respir Crit Care Med, 158(2), 629-634. https://doi.org/10.1164/ajrccm.158.2.9711023

Cadore, E. L., Casas-Herrero, A., Zambom-Ferraresi, F., Idoate, F., Millor, N., Gómez, M., . . . Izquierdo, M. (2014). Multicomponent exercises including muscle power training enhance muscle mass, power output, and functional outcomes in institutionalized frail nonagenarians. Age, 36, 773-785. https://doi.org/10.1007/s11357-013-9586-z

Casas-Herrero, A., Cadore, E. L., Zambom-Ferraresi, F., Idoate, F., Millor, N., Martínez-Ramirez, A., ... Izquierdo, M. (2013). Functional capacity, muscle fat infiltration, power output, and cognitive impairment in institutionalized frail oldest old. Rejuvenation Research, 16, 396-403. https://doi.org/10.1089/rej.2013.1438

Caspersen, C.J., Powell, K.E., \& Christenson, G.M. (1985). Physical activity, exercise and physical fitness: definitions and distinctions for health-related research. Public Health Rep, 100(2), 126-131.

Cebollero, P., Zambom-Ferraresi, F., Hernández, M., Hueto, J., Cascante, J., \& Anton, M.M. (2017). Inspiratory fraction as a marker of skeletal muscle dysfunction in patients with COPD. Rev Port Pneumol, 23(1), 3-9. https://doi.org/10.1016/j.rppnen.2016.07.003

Cebollero, P., Antón, M., Hernández, M. \& Hueto, J. (2018). Walking program for COPD patients: clinical impact after two years of follow-up. Arch Bronconeumol, 54(8), 439-440.

https://doi.org/10.1016/j.arbres.2017.11.002

Coronell, C., Orozco-Levi, M., Méndez, R., Ramírez-Sarmiento, A., Gáldiz, J.B. \& Gea, J. (2004). Relevance of assessing quadriceps endurance in patients with COPD. Eur Respir J, 24(1), 129-136. https://doi.org/10.1183/09031936.04.00079603

Decramer, M., Gosselink, R., Troosters, T., Verschueren, M., \& Evers, G. (1997). Muscle weakness is related to utilization of health care resources in COPD patients. European Respiratory Journal, 10, 417-423. https://doi.org/10.1183/09031936.97.10020417

Garcia-Aymerich, J., Serra, I., Gómez, F.P., Farrero, E., Balcells, E., Rodríguez, D.A., . . Antó, J.M. (2009). Physical activity and clinical and functional 
status in COPD. Chest, 136(1), 62-70. https://doi.org/10.1378/chest.082532

Gosker, H.R., Kubat, B., Schaart, G., Van Der Vusse, G.J., Wouters, E.F. \& Schols, A.M. (2003). Myopathological features in skeletal muscle of patients with chronic obstructive pulmonary disease. Eur Respir J, 22(2):280-285. https://doi.org/10.1183/09031936.03.00012803

Gosselink, R., Troosters, T., \& Decramer, M. (1996). Peripheral muscle weakness contributes to exercise limitation in COPD. American Journal of Respiratory and Critical Care Medicine, 153, 976-980. https://doi.org/10.1164/ajrccm.153.3.8630582

Hernández, M., Zambom-Ferraresi, F., Cebollero, P., Hueto, J., Cascante, J.A. \& Antón, M.M. (2017). The relationships between muscle power and physical activity in older men with chronic obstructive pulmonary disease. J Aging Phys Act, 25(3), 360-366. https://doi.org/10.1123/japa.2016-0144

Hunter, G.R., Mccarthy, J.P. \& Bamman, M.M. (2004). Effects of resistance training on older adults. Sports Med, 34(5), 329-348. https://doi.org/10.2165/00007256-200434050-00005

Hurley, B.F. (1995). Age, gender, and muscular strength. J Gerontol A Biol Sci Med Sci, 50, 41-44. https://doi.org/10.1093/gerona/50A.Special_Issue.41

Izquierdo, M., Ibañez, J., Gorostiaga, E., Garrues, M., Zúñiga, A., Antón, A., . . . \& Häkkinen, K. (1999). Maximal strength and power characteristics in isometric and dynamic actions of the upper and lower extremities in middle-aged and older men. Acta Physiol Scand, 167(1), 57-68. https://doi.org/10.1046/j.1365-201x.1999.00590.x

Leirós-Rodríguez, R., Romo-Pérez, V., Soto-Rodríguez, A. \& García-Soidán, J.L. (2018). Prevalencia de las limitaciones funcionales durante el envejecimiento en la población española y su relación con el índice de masa corporal. Retos, 34, 200-204. https://doi.org/10.47197/retos.v0i34.58958

Lera-López, F., Garrues Irisarri, M.A., Ollo-López, A., Sánchez Iriso, E., Cabasés Hita, J.M. \& Sánchez Santos, J.M. (2017). Actividad física y salud autopercibida en personas mayores de 50 años. Revista Internacional de Medicina y Ciencias de la Actividad Física y el Deporte, 17(67), 559-571. https://doi.org/10.15366/rimcafd2017.67.011

Maltais, F., Decramer, M., Casaburi, R., Barreiro, E., Burelle, Y., Debigaré, R., .. Wagner, P.D. (2014). An official American Thoracic Society/European Respiratory Society statement: update on limb muscle dysfunction in chronic obstructive pulmonary disease. Am J Respir Crit Care Med, 189(9), e15-62. https://doi.org/10.1164/rccm.201402-0373ST

Marquis, K., Debigaré, R., Lacasse, Y., LeBlanc, P., Jobin, J., Carrier, G., \& Maltais, F. (2002). Midthigh muscle cross-sectional area is a better predictor of mortality than body mass index in patients with chronic obstructive pulmonary disease. American Journal of Respiratory and Critical Care Medicine, 166, 809-813. https://doi.org/10.1164/rccm.2107031

Miravitlles, M., Soriano, J.B., García-Río, F., Muñoz, L., Duran-Tauleria, E., Sanchez, G., . . Ancochea, J. (2009). Prevalence of COPD in Spain: impact of undiagnosed COPD on quality of life and daily life activities. Thorax, 64(10), 863-868. https://doi.org/10.1136/thx.2009.115725 
Moral-García, J.E., Al Nayf Mantas, M.R., López-García, S., Maneiro, R. \& Amatria, M. (2019). Estado nutricional y condición física en personas mayores activas vs. Sedentarias. Revista Internacional de Medicina y Ciencias de la Actividad Física y el Deporte, 9(76), 685-698. https://doi.org/10.15366/rimcafd2019.76.003

Mostert, R., Goris, A., Weling-Scheepers, C., Wouters, E.F. \& Schols, A.M. (2000). Tissue depletion and health related quality of life in patients with chronic obstructive pulmonary disease. Respir Med, 94(9), 859-867. https://doi.org/10.1053/rmed.2000.0829

Nyberg, A., Saey, D., \& Maltais, F (2015). Why and how limb muscle mass and function should be measured in patients with chronic obstructive pulmonary disease. Ann Am Thorac Soc, 12(9), 1269-1277. doi: 10.1513/AnnalsATS.201505-278PS

Seymour, J. M., Spruit, M. A., Hopkinson, N. S., Natanek, S. A., Man, W. D., Jackson, A., ... Wouters, E. F. (2010). The prevalence of quadriceps weakness in COPD and the relationship with disease severity. European Respiratory Journal, 36(1), 81-88. https://doi.org/10.1183/09031936.00104909

Salinas Martínez, F.S., Cocca, A., Mohamed, K., \& Ramírez, J.V. (2010). Actividad física y sedentarismo: repercusiones sobre la salud y calidad de vida de las personas mayores. Retos, 17, 126-129.

Swallow, E.B., Reyes, D., Hopkinson, N.S., Man, W.D., Porcher, R., Cetti, E,J., . . . Polkey, M.I. (2007). Quadriceps strength predicts mortality in patients with moderate to severe chronic obstructive pulmonary disease. Thorax, 62(2), 115-120. https://doi.org/10.1136/thx.2006.062026

van de Bool, C., Rutten, E.P.A., van Helvoort, A., Franssen, F.M.E., Wouters, E.F.M., \& Schols, A.M.W.J. (2017). A randomized clinical trial investigating the efficacy of targeted nutrition as adjunct to exercise training in COPD. $J$ Cachexia Sarcopenia Muscle, 8(5), 748-758. https://doi.org/10.1002/jcsm.12219

Vestbo, J., Hurd, S. S., Agustí, A. G., Jones, P. W., Vogelmeier, C., Anzueto, A., . . Rodriguez-Roisin, R. (2013). Global strategy for the diagnosis, management, and prevention of chronic obstructive pulmonary disease: Gold executive summary. American Journal of Respiratory and Critical Care Medicine, 187, 347-365. https://doi.org/10.1164/rccm.2012040596PP

Waschki, B., Kirsten, A.M, Holz, O., Mueller, K.C., Schaper, M., Sack, A.L., . . . Watz, H. (2015). Disease progression and changes in physical activity in patients with chronic obstructive pulmonary disease. Am J Respir Crit Care Med, 192(3):295-306. https://doi.org/ 10.1164/rccm.201501-00810C

Zambom-Ferraresi, F., Cebollero, P., Gorostiaga, E.M., Hueto, J., Cascante, J., ... Anton, M.M. (2015). Effects of combined resistance and endurance training versus resistance training alone on strength, exercise capacity, and quality of life in patients with COPD. J Cardiopulm Rehabil Prev, 35(6):446-453. https://doi.org/10.1097/HCR.0000000000000132

Número de citas totales / Total references: 34 (100\%) 
Rev.int.med.cienc.act.fís.deporte - vol. 21 - número 82 - ISSN: 1577-0354

Número de citas propias de la revista / Journal's own references: 2 (5.9\%)

Rev.int.med.cienc.act.fís.deporte- vol. X - número X - ISSN: 1577-0354 